\title{
Contraction limits of the proton-neutron symplectic model
}

\author{
H. G. Ganev1,a b \\ ${ }^{1}$ Bogoliubov Laboratory of Theoretical Physics, JINR, Dubna, Moscow region, 141980 Russia
}

\begin{abstract}
The algebraic approach to nuclear structure physics allows a certain microscopic collective motion algebra to be also interpreted on macroscopic level which is achieved in the limit of large representation quantum numbers. Such limits are referred to as macroscopic or hydrodynamic limits and show how a given microscopic discrete system starts to behave like a continuous fluid. In the present paper, two contraction limits of the recently introduced fully microscopic proton-neutron symplectic model (PNSM) with the $\operatorname{Sp}(12, R)$ dynamical symmetry algebra are considered. As a result, two simplified macroscopic models of nuclear collective motion are obtained in simple geometrical terms. The first one is the $U(6)$-phonon model with the semi-direct product structure $[H W(21)] U(6)$, which is shown to be actually an alternative formulation of the original proton-neutron symplectic model in the familiar IBM-terms. The second model which appears in double contraction limit is the two-rotor model with the $R O T_{p}(3) \otimes R O T_{n}(3) \supset R O T(3)$ algebraic structure. The latter, in contrast to the original two-rotor model, is not restricted to the case of two coupled axial rotors. In this way, the second contraction limit of the PNSM, provides the phenomenological two-rotor model with a simple microscopic foundation.
\end{abstract}

\section{Introduction}

Symmetry is an important concept in physics. In finite many-body systems, it appears as time reversal, parity, and rotational invariance, but also in the form of dynamical symmetries [1-6]. The standard symmetry approach allows the construction of a Hamiltonian of a system under consideration which is, or nearly so, invariant under a group of symmetry transformations. Group theory then allows one to construct basis states realizing the symmetry and explicit matrix elements for physically interesting transition operators themselves classified by the symmetry. Many properties of atomic nuclei $[1,3-6]$ have been investigated using algebraic models, in which one obtains bands of collective states which span irreducible representations of the corresponding dynamical groups.

The algebraic approach in nuclear structure physics is also particularly useful because a certain algebra can often be interpreted both at the macroscopic (hydrodynamic) and microscopic levels. There is a formal expansioncontraction group-theoretical procedure [7-9] which allows to accomplish such a relation between the microscopic and macroscopic aspects of a given many-particle nuclear system. Contraction limits are obtained at large representations of the microscopic collective algebras under consideration. In this way, at large quantum numbers, one obtains the macroscopic or hydrodynamic limits which show how a discrete microscopic system starts to behave like a continuous hydrodynamic system. That

\footnotetext{
ae-mail: huben@theor.jinr.ru

b Permanent address: Institute of Nuclear Research and Nuclear Energy, Bulgarian Academy of Sciences, Sofia, Bulgaria
}

is why such limits are referred to as hydrodynamic or many-particle limits. Through the exploration of macroscopic limits new models appear in simple geometrical terms which reveal the underlying physical dynamics of the original algebraic models.

Recently, the fully microscopic proton-neutron symplectic model (PNSM) of nuclear collective motion was introduced by considering the symplectic geometry and possible collective flows in the two-component many-particle nuclear system [10]. Further, it was shown that, in its hydrodynamic limit, it reduces to the $U(6)$-phonon model with the semi-direct product structure $[H W(21)] U(6)$ which unifies both the two-fluid irrotational-flow collective model and a microscopically based $U(6)$ model [11]. The latter naturally generalizes the $S U(3)$ model of Elliott [12] for the case of two-component many-particle nuclear system and is related to the valence proton-neutron degrees of freedom. From the hydrodynamic perspective, the $U(6)$-phonon model therefore includes the irrotational collective flows and their coupling to the intrinsic vortex degrees of freedom. The latter is of vital importance for the appearance of the low-lying collective bands. In this way the extra degrees of freedom contained in this larger $U(6)$ algebraic structure will therefore embrace the basic $S U(3)$ rotor as well as the low-lying vibrational degrees of freedom.

The appearance of an $U(6)$ intrinsic structure in both the PNSM and $U(6)$-phonon model is of significant importance for the microscopic theory of nuclear collective excitations. In this regard, we recall that the popular Interacting Boson Model [13] has clearly demonstrated that simple algebraic ways exist to get collective spectra within 
$U(6)$-based scheme. Then, within the framework of both the PNSM and $U(6)$-phonon model, the full range of lowlying states could be described by microscopically based $U(6)$ structure along the lines of the IBM, albeit in contrast to the latter, renormalized by their coupling to the giant resonance vibrations. This result could not be overestimated recalling also that in order to obtain the low-lying excited collective bands (e.g., beta band) within the framework of the one-component symplectic model [14] one needs to involve a representation mixing caused by, e.g., pairing, spin-orbit and other symplectic-breaking components of the nuclear interaction (cf. Ref.[15]).

In the present contribution, we show that the $U(6)$ phonon model represents actually an alternative formulation of the PNSM in the familiar IBM-terms. For this purpose, the relevant representation theory of the $U(6)$ phonon model is shortly considered. It is shown that it coincides with that of the original proton-neutron symplectic model [16]. The relation of the $U(6)$-phonon model irreps with the shell-model classification of the basis states is considered by reducing the $U(6)$ group to the direct product space of $S U_{p}(3) \otimes S U_{n}(3)$ irreps, generalizing in this way the Elliott's $S U(3)$ model [12] for the case of twocomponent nuclear system. The physical content of the intrinsic substructure $S U_{p}(3) \otimes S U_{n}(3) \supset S U(3)$ of $U(6)$, associated with the proton-neutron valence shell degrees of freedom, is exposed further by considering its contraction limit. It is shown that, at large representation quantum numbers, it reduces to that of two coupled rigid rotors (two-rotor model) $R O T_{p}(3) \otimes R O T_{n}(3) \supset \operatorname{ROT}(3)$. The latter, in contrast to the original two-rotor model (TRM) [17], is not restricted to the case of two coupled axial rotors. In this way, the second contraction limit of the PNSM, provides the phenomenological TRM with a simple microscopic foundation.

\section{The proton-neutron symplectic model}

Collective observables of the proton-neutron symplectic model, which span the $\operatorname{Sp}(12, R)$ algebra, are given by the following one-body operators [10]:

$$
\begin{aligned}
& Q_{i j}(\alpha, \beta)=\sum_{s=1}^{m} x_{i s}(\alpha) x_{j s}(\beta), \\
& S_{i j}(\alpha, \beta)=\sum_{s=1}^{m}\left(x_{i s}(\alpha) p_{j s}(\beta)+p_{i s}(\alpha) x_{j s}(\beta)\right),(2) \\
& L_{i j}(\alpha, \beta)=\sum_{s=1}^{m}\left(x_{i s}(\alpha) p_{j s}(\beta)-x_{j s}(\beta) p_{i s}(\alpha)\right),(3) \\
& T_{i j}(\alpha, \beta)=\sum_{s=1}^{m} p_{i s}(\alpha) p_{j s}(\beta),
\end{aligned}
$$

where $i, j=1,2,3 ; \alpha, \beta=p, n$ and $s=1, \ldots, m=A-1$. In Eqs. (1)-(4), $x_{i s}(\alpha)$ and $p_{i s}(\alpha)$ denote the coordinates and corresponding momenta of the translationally-invariant Jacobi vectors of the $m$-quasiparticle two-component nuclear system and $A$ is the number of protons and neutrons. By considering the $m$ Jacobi quasiparticles instead of $A$ pro- tons and neutrons, the problem of center-of-mass motion is avoided from the very beginning.

Dynamical content of the PNSM is revealed by considering different dynamical groups that can be constructed from the symplectic operators which generate different classical collective motions, including a wide class of both the in-phase (isoscalar) and out-of-phase (isovector) excitations of the proton subsystem with respect to the neutron one, as well as collective excitations of the combined proton-neutron system as a whole. Thus, the $\operatorname{Sp}(12, R)$ group provides a quite general framework for the investigation of the nature of classical collective motions in nuclei. The PNSM appears as a hydrodynamic collective model of the proton-neutron nuclear system which include 21 collective irrotational-flow degrees of freedom and an $U(6)$ intrinsic structure associated with the vortex degrees of freedom [10].

To quantize the model one has to construct the irreducible representations of the $\operatorname{Sp}(12, R)$ group, appropriate to the many-particle system. Thus, the model space is spanned by the irreducible representations of the $\operatorname{Sp}(12, R)$ algebra. In terms of the harmonic oscillator creation and annihilation operators

$$
\begin{aligned}
& b_{i \alpha, s}^{\dagger}=\sqrt{\frac{m_{\alpha} \omega}{2 \hbar}}\left(x_{i s}(\alpha)-\frac{i}{m_{\alpha} \omega} p_{i s}(\alpha)\right), \\
& b_{i \alpha, s}=\sqrt{\frac{m_{\alpha} \omega}{2 \hbar}}\left(x_{i s}(\alpha)+\frac{i}{m_{\alpha} \omega} p_{i s}(\alpha)\right),
\end{aligned}
$$

the many-particle realization of the $\operatorname{Sp}(12, R)$ Lie algebra is given by [16]:

$$
\begin{aligned}
& F_{i j}(\alpha, \beta)=\sum_{s=1}^{m} b_{i \alpha, s}^{\dagger} b_{j \beta, s}^{\dagger}, \\
& G_{i j}(\alpha, \beta)=\sum_{s=1}^{m} b_{i \alpha, s} b_{j \beta, s}, \\
& A_{i j}(\alpha, \beta)=\frac{1}{2} \sum_{s=1}^{m}\left(b_{i \alpha, s}^{\dagger} b_{j \beta, s}+b_{j \beta, s} b_{i \alpha, s}^{\dagger}\right) .
\end{aligned}
$$

The number-conserving operators $(8)$ generate the maximal compact subgroup $U(6)$ of $\operatorname{Sp}(12, R)$.

From the shell-model perspective, the PNSM appears as a natural multi-major-shell extension of the generalized proton-neutron $S U(3)$ scheme which takes into account the core collective excitations of monopole and quadrupole, as well as dipole type associated with the giant vibrational degrees of freedom [16]. Each $\operatorname{Sp}(12, R)$ irreducible representation is determined by a symplectic bandhead or an intrinsic $U(6)$ space which can be fixed by the underlying proton-neutron shell-model structure, so the theory becomes completely compatible with the Pauli principle. Moreover, the intrinsic $U(6)$ structure is of vital importance for the appearance of the low-lying collective bands.

Further, at large $\operatorname{Sp}(12, R)$ representations, as will see in the next section, the algebraic structure of the protonneutron symplectic model simplifies as a result of the group contraction of its underlying dynamical symmetry algebra. 


\section{Contraction of the $\operatorname{Sp}(12, R)$ algebra}

We consider first the $\operatorname{sp}(2, R) \approx s u(1,1)$ subalgebra. The $s p(2, R)$ generators are obtained from the $\operatorname{Sp}(12, R)$ ones by summation with respect to both indices $i$ and $\alpha$ [11]

$$
\begin{aligned}
K_{+} & =\frac{1}{2} \sum_{i s \alpha} b_{i \alpha, s}^{\dagger} b_{i \alpha, s}^{\dagger}=\frac{1}{2} \sum_{i \alpha} F_{i i}(\alpha, \alpha)=\frac{1}{2} F^{0} \\
K_{-} & =\frac{1}{2} \sum_{i s \alpha} b_{i \alpha, s} b_{i \alpha, s}=\frac{1}{2} \sum_{i \alpha} G_{i i}(\alpha, \alpha)=\frac{1}{2} G^{0},(10) \\
K_{0} & =\frac{1}{4} \sum_{i s \alpha}\left(b_{i \alpha, s}^{\dagger} b_{i \alpha, s}+b_{i \alpha, s} b_{i \alpha, s}^{\dagger}\right) \\
& =\frac{1}{2} \sum_{i \alpha} A_{i i}(\alpha, \alpha)=\frac{1}{2} A^{0}
\end{aligned}
$$

They obey the following commutation relations:

$$
\left[K_{0}, K_{ \pm}\right]= \pm K_{ \pm}, \quad\left[K_{+}, K_{-}\right]=-2 K_{0}
$$

which differ from the standard angular momentum $s u(2)$ commutation relations only by the sign on the right hand side of the last commutator, and are defining for the $s u(1,1)$ algebra, locally isomorphic to $s p(2, R)$ algebra. Note that the operator $2 K_{0}$ is the harmonic oscillator Hamiltonian in units $\hbar \omega$ with eigenvalue $\sigma_{0}=\left(\sigma_{1}+\ldots+\right.$ $\left.\sigma_{6}\right)+\frac{6}{2}(A-1)$.

From the commutation relations (12) one readily obtains [11]

$$
\left[\frac{K_{-}}{\sqrt{\sigma_{0}}}, \frac{K_{+}}{\sqrt{\sigma_{0}}}\right]=I+D,
$$

where the operator $D$ is defined as $D=\left(2 K_{0}-\sigma_{0} I\right) / \sigma_{0}$ and $I$ is the identity operator. For a harmonic oscillator shellmodel state of excitation energy $2 v \hbar \omega$ above the lowestweight state $|\sigma\rangle$, the operator $D$ has eigenvalue $2 v / \sigma_{0}$, which in the limit $\sigma_{0} \rightarrow \infty$ (hydrodynamic limit) tends to 0 . In practice one needs only the condition $2 v<<\sigma_{0}$. The $U(6)$ lowest-weight state $\left[\sigma_{1}, \ldots, \sigma_{6}\right]$ which defines the $\operatorname{Sp}(12, R)$ irreducible representation can be fixed by the underlying proton-neutron shell-model structure. For example, the shell model considerations give the $U(6)$ irrep $[132,86,70,70,70,70]$ for ${ }^{154} \mathrm{Sm}$. From the latter one finds that the minimum number of oscillator quanta allowed by the Pauli principle is $\sigma_{0}=957$. So, the condition $2 v<<\sigma_{0}$ is satisfied for small $v$.

Thus, in the limit $2 v<<\sigma_{0}$, one obtains

$$
\left[\frac{K_{-}}{\sqrt{\sigma_{0}}}, \frac{K_{+}}{\sqrt{\sigma_{0}}}\right]=I
$$

which can be written in terms of the $F$ and $G$ operators (9) and (10)

$$
\left[\frac{G^{0}(p, p)+G^{0}(n, n)}{2 \sqrt{\sigma_{0}}}, \frac{F^{0}(p, p)+F^{0}(n, n)}{2 \sqrt{\sigma_{0}}}\right]=I .
$$

From the latter it follows that the operator $\frac{1}{2 \sqrt{\sigma_{0}}}\left[F^{0}(p, p)+\right.$ $\left.F^{0}(n, n)\right]$ acts like a composite $s$-boson creation operator $s^{\dagger}=s_{p}^{\dagger}+s_{n}^{\dagger}$. Then the commutation relation (15) is satisfied if the following correspondence is made

$$
\begin{array}{ll}
F^{0}(p, p) \leftrightarrow \sqrt{2 \sigma_{0}} s_{p}^{\dagger}, & G^{0}(p, p) \leftrightarrow \sqrt{2 \sigma_{0}} s_{p}, \\
F^{0}(n, n) \leftrightarrow \sqrt{2 \sigma_{0}} s_{n}^{\dagger}, & G^{0}(n, n) \leftrightarrow \sqrt{2 \sigma_{0}} s_{n} .
\end{array}
$$

Equation (16) together with $A^{0} \leftrightarrow A^{0}$ establish a relation between the $s p(2, R) \approx s u(1,1) \subset s u_{p}(1,1) \oplus s u_{n}(1,1)$ and $u(1)$-phonon algebras. The obtained correspondence is known also as a contraction limit. In a similar manner, one can obtain the correspondence between the remainder of the $\operatorname{Sp}(12, R)$ and $U(6)$-phonon algebras. Thus, the full correspondence is given by [11]:

$$
\begin{aligned}
& F^{0}(p, p) \leftrightarrow \sqrt{2 \sigma_{0}} s_{p}^{\dagger}, \quad F_{M}^{2}(p, p) \leftrightarrow \sqrt{2 \sigma_{0}} d_{M, p}^{\dagger}, \\
& F^{0}(n, n) \leftrightarrow \sqrt{2 \sigma_{0}} s_{n}^{\dagger}, \quad F_{M}^{2}(n, n) \leftrightarrow \sqrt{2 \sigma_{0}} d_{M, n}^{\dagger}, \\
& F^{0}(p, n) \leftrightarrow \sqrt{2 \sigma_{0}} s_{\delta}^{\dagger}, \quad F_{M}^{2}(p, n) \leftrightarrow \sqrt{2 \sigma_{0}} d_{M, \delta}^{\dagger}, \\
& F_{M}^{1}(p, n) \leftrightarrow \sqrt{2 \sigma_{0}} p_{M, \delta}^{\dagger}, \quad A_{M}^{L}(\alpha, \beta) \leftrightarrow A_{M}^{L}(\alpha, \beta),
\end{aligned}
$$

and their hermitian conjugate counterparts. The set of operators $\left\{s_{\tau}^{\dagger}, d_{M, \tau}^{\dagger}, p_{M, \delta}^{\dagger}, s_{\tau}, d_{M, \tau}, p_{M, \delta}, I\right\}(\tau=p, n, \delta)$ generates the $h w(21)$ phonon algebra. The later consists of the IBM-3 building blocks [13] plus the extra degrees of freedom represented by the components of the dipole $p$-boson. From the above correspondence, however, we see that in contrast to the IBM case in which the $s$ and $d$ bosons represent correlated valence fermion pairs, in the hydrodynamic limit of the $\operatorname{Sp}(12, R)$ model, the $s$ and $d$ bosons are in fact superpositions of pairs of harmonic oscillator vector excitations (phonons) (cf. Eqs.(6)-(7)) which represent multi-major-shell collective core excitations.

\section{The $U(6)-p h o n o n$ and two-rotor models}

\subsection{The $U(6)$-phonon model}

The phonon and $u(6)$ operators together span a semi-direct sum Lie algebra $[h w(21)] u(6)$ which we call $u(6)$-phonon algebra. Then, the $U(6)$-phonon model is a model whose state space carries an unitary irreducible representation of the $u(6)$-phonon algebra and for which the Hamiltonian and collective observables are simple functions of the $u(6)$ and phonon operators.

If we identify the $\pm 2 \hbar \omega$ phonon operators of the $h w(21)$ algebra with those of the two-fluid irrotationalflow model, then it is clear that the PNSM contains the former as a submodel. However, it is also clear from the microscopic realization of the $s p(12, R)$ algebra that the additional $u(6)$ intrinsic degrees of freedom appear which turn out to be crucial for the appearance of low-lying collective bands. From the general linear flow origins of the symplectic model, we know that these extra degrees of freedom are associated with the internal (vortex) motions [10]. Among the latter are the vortex spin rotations. In this way, from the hydrodynamic perspective, the $U(6)$ phonon model is an extension of the two-fluid irrotationalflow collective model to include the intrinsic vortex degrees of freedom. 
An $U(6)$-phonon irreducible representation is defined by the $U(6)$ highest-weight state $|\sigma\rangle$ with $\sigma=\left[\sigma_{1}, \ldots, \sigma_{6}\right]$ which is also a phonon vacuum, i.e. satisfies the following equations

$$
\begin{aligned}
& u|\sigma\rangle=0, \\
& A_{a b}|\sigma\rangle=0, \quad a<b \\
& A_{a a}|\sigma\rangle=\left(\sigma_{a}+\frac{1}{2}(A-1)\right)|\sigma\rangle
\end{aligned}
$$

with $\sigma_{0}=\sigma_{1}+\ldots+\sigma_{6}+\frac{6}{2}(A-1)$, where $a, b=1, \ldots, 6$ and $u$ represents any annihilation operator of the $h w(21)$ algebra. Note that the $u(6)$-phonon algebra is non-compact and hence its unitary irreducible representations are infinite dimensional and have no highest weights. Thus its irreps are defined by the lowest-weight states which are also $U(6)$ highest-weight states. Such an $U(6)$ irrep whose highest-weight state is also an $U(6)$-phonon lowest-weight state is said to be a lowest-grade $U(6)$ irrep for that $U(6)$ phonon irrep. The other states of the basis are then generated by repeated action of the phonon raising operators on the $U(6)$ irreducible states. The phonon operators $u^{\dagger} \equiv\left\{s_{\tau}^{\dagger}, d_{M, \tau}^{\dagger}, p_{M, \delta}^{\dagger}\right\}$ carry the [2] representation of $U(6)$. Then, the $U(6)$-phonon basis can be classified according to the chain [11]:

$$
\begin{aligned}
& {[H W(21)] U(6) \supset U(6)} \\
& \sigma \quad n \rho \quad E
\end{aligned}
$$

and written in the form

$$
|\sigma n \rho E \eta\rangle=\left[Z^{(n)}\left(u^{\dagger}\right) \times|\sigma\rangle\right]_{\eta}^{\rho E},
$$

with $U(6)$ quantum numbers $n=\left[n_{1}, \ldots, n_{6}\right]$ running over the sets of even integers for which $n_{1} \geq n_{2} \geq \ldots \geq n_{6} \geq 0$ and $n_{1}+\ldots+n_{6}=2 N$. In (22) the square brackets denote an $U(6)$-coupling of the polynomial $Z^{(n)}\left(u^{\dagger}\right)$ with the intrinsic $U(6)$ structure $|\sigma\rangle, \rho$ is a multiplicity index and $\eta$ denotes a basis for the coupled $U(6)$ irrep $E$. The latter can be fixed by considering different subgroup chains which reduce the $U(6)$ to the rotational group $S O(3)$. The shell-model considerations suggest the reduction [16]:

$$
\begin{array}{lll}
U(6) & \supset S U_{p}(3) \otimes S U_{n}(3) \\
E & \gamma \quad\left(\lambda_{p}, \mu_{p}\right)\left(\lambda_{n}, \mu_{n}\right) \\
& & \\
& \supset S U(3) \supset S O(3) \supset S O(2) . \\
& \varrho(\lambda, \mu) K \quad L \quad M
\end{array}
$$

The chain (23) naturally generalizes the Elliot $S U(3)$ model [12] by extending the model space to the direct product space $S U_{p}(3) \otimes S U_{n}(3)$ of proton and neutron subsystems. The $S U(3)$ irreps of the two subsystems are subsequently coupled to the $S U(3)$ irrep of the combined proton-neutron system. The chain (23) corresponds to the following choice of the index $\eta=$ $\gamma\left(\lambda_{p}, \mu_{p}\right)\left(\lambda_{n}, \mu_{n}\right) \varrho(\lambda, \mu) K L M$ labeling the basis states (22). In this way one obtains a representation theory which is precisely the same as that given in Ref. [16].

The phonon structure of the $[H W(21)] U(6)$ model therefore enables it to simultaneously include the giant
Table 1. The $U(6)$-phonon representation of ${ }^{154} \mathrm{Sm}$ defined by the lowest-grade $U(6)$ irrep $[132,86,70,70,70,70] \equiv[62,16]$.

$[66,16],[65,17],[65,16,1], 2[64,18], 2[64,17,1]$,

$2[64,16,2], 2[63,19], 3[63,18,1],[63,17,2]$,

$2[62,20], 3[62,19,1], 3[62,18,2],[62,18,1,1]$,

$3[62,17,3],[62,17,2,1], 3[62,16,4],[62,16,3,1]$,

$[62,16,2,2]$

$[64,16],[63,17],[63,16,1],[62,18],[62,16,2]$,

$[62,16,1,1]$

$[62,16]$

monopole and quadrupole, as well as dipole resonances into nuclear collective dynamics. On the other hand, the low-lying rotational bands are associated primarily with the proton-neutron valence shell degrees of freedom represented by the intrinsic $U(6)$ structure.

If the $U(6)$-phonon lowest-weight state $|\sigma\rangle$ reduces to the phonon vacuum $|0\rangle$, then one obtains a 21 -dimensional two-fluid irrotational-flow collective model. Thus, by allowing the phonon vacuum to have a non-trivial intrinsic $U(6)$ structure gives the collective model a much richer structure and a framework for considering the low-lying collective bands in an unified manner. This result is of significant importance for the theory of nuclear collective excitations.

As an illustration, the $U(6)$-phonon irreducible representation of ${ }^{154} \mathrm{Sm}$, determined by the lowest-grade $U(6)$ irrep $[132,86,70,70,70,70]$, is given in Table 1. As we can see from it, the relevant $U(6)$ irreps comprising the $U(6)$-phonon irreducible representation under consideration are not fully symmetric. However, one expects the most symmetric $U(6)$ irreps $E$ to be dominant in the lowenergy spectra of the heavy deformed even-even nuclei. The classification of the $S U(3)$ basis states for ${ }^{154} \mathrm{Sm}$ according to the decompositions given by the chain (23) for the $U(6)$-phonon irreducible representation under consideration, restricted to the most symmetric two-rowed $U(6)$ partitions, is given in Table 2. From the latter the structure of the basis becomes evident. The $S U(3)$ basis states so obtained are precisely those which can be obtained respectively by acting on the intrinsic base space states $(\lambda, \mu)$ by the $S U(3)(2,0) s_{\tau}^{\dagger}, d_{M, \tau}^{\dagger}$ and $(0,1) p_{M, \delta}^{\dagger}$ phonon raising operators. We also see repeating of the $S U(3)$ multiplets within different $U(6)$ irreducible representations.

The fact that one obtains a nonscalar $U(6)$-phonon irreducible representation for a particular nucleus is a very important feature of the present approach, which is a consequence of the two-component composite character of the nuclear systems. As we can see from Table 2, the $U(6)$ intrinsic structure (the first row with $2 N=0$ ) contains many $S U$ (3) multiplets which are appropriate for the description of different low-lying collective bands (ground state, $\beta, \gamma$, etc.) in the spectra of heavy even-even deformed nuclei. In this way the $U(6)$ intrinsic structure of the $U(6)$-phonon model provide us with a framework for the simultaneous description of low-lying collective states of well deformed nuclei in a manner similar to that of IBM, but in contrast 
Table 2. The classification of the $S U(3)$ basis states for ${ }^{154} \mathrm{Sm}$.

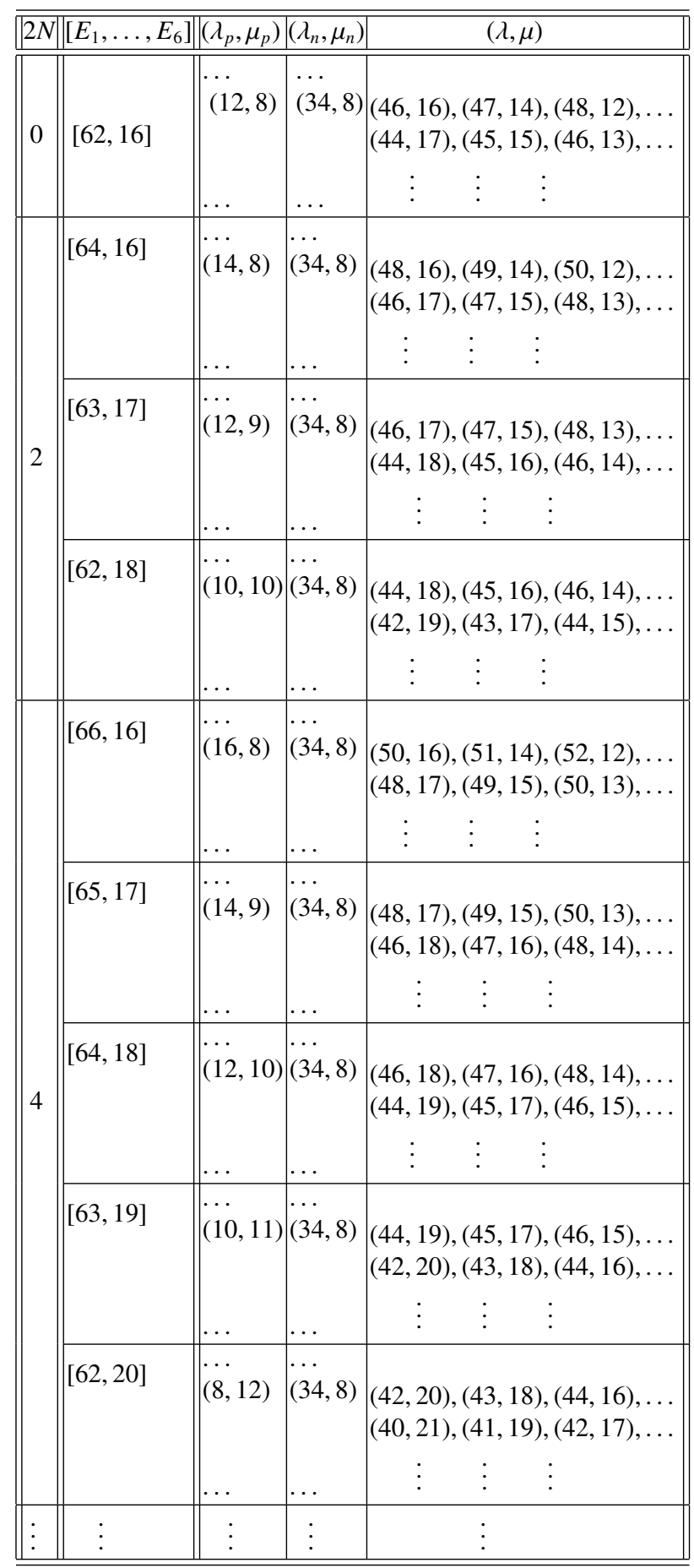

to the latter, with states which are renormalized by their coupling to the giant resonance vibrational degrees of freedom.

\subsection{The coupled two-rotor model}

The combined $s u(3)$ algebra of the proton-neutron system is spanned by the set $\operatorname{su}(3)=\left\{\widetilde{Q}_{M}, L_{M}\right\}$, where $\widetilde{Q}_{M}=$ $\widetilde{Q}_{M}^{p}+\widetilde{Q}_{M}^{n}$ are the truncated (Elliott) quadrupole momentum operators and $L_{M}=L_{M}^{p}+L_{M}^{n}$. The elements of $s u(3)$ satisfy the following commutation relations:

$$
\begin{aligned}
& {\left[L_{M}, L_{M^{\prime}}\right]=-\sqrt{2}\left\langle 1 M, 1 M^{\prime} \mid 1 M+M^{\prime}\right\rangle L_{M+M^{\prime}},} \\
& {\left[L_{M}, \widetilde{Q}_{M^{\prime}}\right]=-\sqrt{6}\left\langle 1 M, 2 M^{\prime} \mid 2 M+M^{\prime}\right\rangle \widetilde{Q}_{M+M^{\prime}},} \\
& {\left[\widetilde{Q}_{M}, \widetilde{Q}_{M^{\prime}}\right]=3 \sqrt{10}\left\langle 2 M, 2 M^{\prime} \mid 1 M+M^{\prime}\right\rangle L_{M+M^{\prime}} .}
\end{aligned}
$$

Now, if we introduce the rescaled mass quadrupole operators $q_{M}=\frac{Q_{M}}{\sqrt{\epsilon}}$, where $\epsilon=\left\langle C_{2}[S U(3)]\right\rangle=4\left(\lambda^{2}+\mu^{2}+\lambda \mu+\right.$ $3 \lambda+3 \mu)$ is the eigenvalue of the second order Casimir operator $C_{2}[S U(3)]=\widetilde{Q} \cdot \widetilde{Q}+3 L^{2}$ of $S U(3)$, for the Eq. (26) one obtains

$$
\left[q_{M}, q_{M^{\prime}}\right]=3 \sqrt{10}\left\langle 2 M, 2 M^{\prime} \mid 1 M+M^{\prime}\right\rangle \frac{L_{M+M^{\prime}}}{\epsilon} \longrightarrow 0,
$$

when $\epsilon \rightarrow \infty$. In this way, we have obtained the well known result that, in the limit of large $s u(3)$ quantum numbers, the $s u(3)$ algebra contracts to that of rigid rotor algebra $\operatorname{rot}(3)=\left[R^{5}\right] \operatorname{so}(3) \equiv\left\{q_{M}, L_{M}\right\}[18]$, where $R^{5}$ is the fivedimensional abelian Lie algebra spanned by the commuting mass quadrupole operators and so(3) is the angular momentum algebra. Similarly, one can obtain the contractions of the proton and neutron $s u(3)$ algebras. Thus, in double contraction limit, we have obtained the subgroup chain:

$$
\begin{aligned}
{[H W(21)] U(6) } & \supset U(6) \supset R_{D} O T_{p}(3) \otimes R O T_{n}(3) \\
& \supset R O T(3) \supset S O(3),
\end{aligned}
$$

which contains the algebraic structure of two coupled rigid rotors (two-rotor model). Note, however, that in contrast to the original TRM [17], the present algebraic structure assumes the more general case of two coupled non-axial rotors. This is obvious even on the $S U(3)$ level. We recall that the reduction of the generic $U(6)$ irreducible representation $E \equiv\left[E_{1}, E_{2}, E_{3}, E_{4}, E_{5}, E_{6}\right]$ to the direct product irreps of $S U_{p}(3) \otimes S U_{n}(3)$ allows irreps of the type $\left(\lambda_{p}, \mu_{p}\right) \otimes\left(\lambda_{n}, \mu_{n}\right)$ with nonzero values of the quantum numbers $\lambda$ and $\mu$ characterizing the proton and neutron $S U(3)$ irreps. The latter geometrically corresponds to two nonaxial rotors [19]. The considered contraction limit gives the phenomenological TRM simple microscopic foundation.

From the shell-model perspective, two-rotor model degrees of freedom correspond to the $S U_{p}(3) \otimes S U_{n}(3) \supset$ $S U(3)$ proton-neutron dynamics of the $0 \hbar \omega$ space. The hydrodynamic limit $\left(\sigma_{0} \rightarrow \infty\right)$ of the symplectic model shows, from the other side, that the underlying vibrational degrees of freedom are associated with the $2 \hbar \omega$ giant resonances of monopole $(L=0)$, quadrupole $(L=2)$ and dipole $(L=1)$ type. Note that the latter is not the giant dipole resonance corresponding to the $1 \hbar \omega$ excitation mode, which however can be easily incorporated into the PNSM by making its central extension, i.e. by considering the semidirect structure $W S p(12, R) \equiv[H W(6)] \operatorname{Sp}(12, R)$ where the $H W(6)$ algebra is spanned by the harmonic oscillator raising and lowering operators (5) together with the identity operator $I$.

Then, in double contraction limit, the states of a single $\operatorname{Sp}(12, R)$ irrep could be identified with the two-rotor 
model states coupled to the 21-dimensional vibrator associated with the giant resonance vibrational degrees of freedom. We note that, from the hydrodynamic perspective, the latter are of irrotational-flow character [10].

\section{Conclusions}

It was shown that the algebraic approach to nuclear structure physics allows a certain microscopic collective motion algebra to be also interpreted on macroscopic level which is achieved in the limit of large representation quantum numbers. Such limits are referred to as macroscopic or hydrodynamic limits and show how a given microscopic discrete system starts to behave like a continuous fluid.

In particular, in the present contribution, two contraction limits of the recently introduced fully microscopic proton-neutron symplectic model with the $\operatorname{Sp}(12, R)$ dynamical symmetry algebra were considered. As a result, two simplified macroscopic models of nuclear collective motion are obtained in simple geometrical terms. The first one is the $U(6)$-phonon model with a semi-direct product structure $[H W(21)] U(6)$, which is shown to be actually an alternative formulation of the original proton-neutron symplectic model in the familiar IBM-terms. The phonon structure of the $[H W(21)] U(6)$ model enables it to simultaneously include the giant monopole and quadrupole, as well as dipole resonances into nuclear collective dynamics. The relation of the $U(6)$-phonon model irreps with the shell-model classification of the basis states is further considered by reducing the $U(6)$ group to the direct product space of $S U_{p}(3) \otimes S U_{n}(3)$ irreps, generalizing in this way the Elliott's $S U$ (3) model for the case of two-component nuclear system. The $S U(3)$ irreps of the two subsystems are subsequently coupled to the $S U(3)$ irrep of the combined proton-neutron system. The $U(6)$ intrinsic structure of the $[H W(21)] U(6)$ model therefore gives a framework for the simultaneous description of the ground state band, as well as the other excited low-lying collective bands.

At large quantum numbers, the intrinsic substructure $S U_{p}(3) \otimes S U_{n}(3) \supset S U(3)$ of $U(6)$, associated with the proton-neutron valence shell degrees of freedom, further reduces to $\operatorname{ROT}_{p}(3) \otimes R O T_{n}(3) \supset R O T(3)$, i.e. to that of two coupled rigid rotors (two-rotor model). In this way, in double contraction limit, the $s p(12, R)$ algebra reduces to the coupled two-rotor model algebra $\operatorname{rot}_{p}(3) \oplus \operatorname{rot}_{n}(3) \supset$ $\operatorname{rot}(3)$ and a phonon algebra $h w(21)$ of the giant resonance vibrational degrees of freedom. The full range of lowlying collective states could then be described as two-rotor model states, renormalized by their coupling to the giant resonance vibrations.

\section{References}

[1] Dynamical Groups and Spectrum Generating Algebras, Vol. 1 and 2, eds. A. Bohm, Y. Ne'eman, and A. O. Barut (World Scientific Publishing Co. Pte. Ltd., Singapore, 1988)

[2] F. Iachello, Lie Algebras and Applications, Lect. Notes Phys. 708 (Springer-Verlag, Berlin Heidelberg, 2006)

[3] Algebraic Approaches to Nuclear Structure: Interacting Boson and Fermion Models, ed. R.F. Casten (Harwood Academic Publishers, New York, 1993)

[4] A. Frank, P.V. Isacker, J. Jolie, Symmetries in Atomic Nuclei: From Isospin to Supersymmetry, Springer Tracks in Modern Physics 230 (Springer-Verlag, New York, 2009)

[5] D.J. Rowe and J.L. Wood, Fundamentals of $\mathrm{Nu}$ clear Models: Foundational Models (World Scientific Publisher Press, Singapore, 2010)

[6] F. Iachello and P. Van Isacker, The Interacting BosonFermion Model (Cambridge University Press, Cambridge, 1991)

[7] E. İnönü and E.P. Wigner, Proc. Nat. Acad. 39, 510 (1953)

[8] E. İnönü and E.P. Wigner, Proc. Nat. Acad. 40, 119 (1954)

[9] R. Gilmore, Lie Groups, Lie Algebras and Some of Their Applications (Wiley, New York, 1974)

[10] H.G. Ganev, Eur. Phys. J. A 50, 183 (2014)

[11] H. G. Ganev, Int. J. Mod. Phys. E 24, 1550039 (2015)

[12] J.P. Elliott, Proc. R. Soc. London, Ser. A 245, 128 (1958); 245, 562 (1958)

[13] F. Iachello and A. Arima, The Interacting Boson Model (Cambridge University Press, Cambridge, 1987)

[14] D.J. Rowe and G. Rosensteel, Phys. Rev. Lett. 38, 10 (1977)

[15] J. Carvalho et al., Nucl. Phys. A 452, 240 (1986)

[16] H.G. Ganev, Eur. Phys. J. A 51, 84 (2015)

[17] N. Lo Iudice and F. Palumbo, Phys. Rev. Lett. 41, 1532 (1978); N. Lo Iudice and F. Palumbo, Nucl. Phys. A 326, 193 (1979)

[18] D.J. Rowe, R. Le Blanc, and J. Repka, J. Phys. A: Math. Gen. 22, L309 (1989)

[19] O. Castanos, J.P. Draayer, and Y. Leschber, Ann. Phys. 180, 290 (1987) 